\title{
KNOWLEDGE BASED CLASSIFIER BASED ON BACKSCATTERING COEFFICIENT FOR MONITORING THE CROP GROWTH ANALYSIS USING MULTI-TEMPORAL IMAGES OF SPACE-BORNE SYNTHETIC APERTURE RADAR (SAR) SENSORS
}

\author{
A. K. Verma ${ }^{1, *}$, R. Nandan ${ }^{2}$, and A. Verma ${ }^{3}$ \\ ${ }^{1}$ Vidyadaan Institute of Technology and Management, Aryabhatta Knowledge \\ University, Dumraon- 802119, Bihar, India - arun@vidyadaan.org \\ ${ }^{2}$ Geology Department, B.N. College, Patna University, Patna-800020, Bihar, India - nandanrranbir@gmail.com \\ ${ }^{3}$ Qualcomm India Pvt Ltd, Raheja Mindspace IT Park, Madhapur, Hyderabad- 500081, Telangana, India - \\ aditi.verma@gmail.com
}

Commission III, WG III/10

KEY WORDS: Backscattering Coefficients, Synthetic Aperture Radar, Crop Growth Analysis, Multi-Temporal Images

\begin{abstract}
:
Space-based observation of crops and agro-system on the Earth surface is one of the most important applications of remote sensing using the sensors in optical and microwave spectrum to assess the crop growth for decision making for developing crop information and management system. Remote sensing technology provides scalable and reliable information in respect of rice crop grown area, its crop growth and prediction of crop yield due to acquisition of satellite imagery during the revisit of the orbit by space-borne sensors in optical and microwave spectrum. Synthetic Aperture Radar has the advantages of all-weather, day and night imaging, canopy penetration, and high-resolution capabilities, which makes Space-borne SAR sensors as an effective system for monitoring crop growth, crop classification and mapping of crop area based on the crop canopy interaction of SAR signals due to backscattering coefficients of the earth surface. SAR data from ERS-1/2 SAR, ENVISAT ASAR, ALOS-1/2 PALSAR, Radarsat-1/2 SAR, TerraSAR, COSMO-SkyMed, and Sentinel-1 have been used by various researchers for identification and analysis of rice crop growth based on the backscattering values in different regions of Asia and European region, where backscattered image depends of various earth surface and SAR sensors parameters. In this paper, knowledge based classifier using SAR images of existing space-borne-SAR sensors have been developed based on modeling of SAR backscattering coefficients in C-band and X-band for monitoring the rice crop growth and its analysis using multi-temporal and multi-frequency- SAR sensors data.
\end{abstract}

\subsection{INTRODUCTION}

Asian countries are the major rice and wheat growing region of the world due to its summer seasons with monsoon rainfall followed by winter seasons. Rice is considered as a pivotal political commodity in many Asian countries with its price often serves as a key indicator for government performance and crucial for policymakers to control rice trade flow for domestic rice market stable, which requires reliable information on rice area, seasonality, and yield as an essential part of many countries of Asian region in the context of food security and policy. This information forms the basis of policy decisions related to imports, exports and prices, which directly impact food security, especially amongst the poor. Normally, rice plants are transplanted in the paddy fields under flooded conditions and irrigated continuously until the mid-maturing stage of rice crops, so that the soil surface of paddy fields is under flooded conditions during the most growing period. The soil surface is kept smooth and fully saturated with water to maintain highly homogenous paddy fields during early vegetative and mid-maturing stages. However, there are technical challenges in the development of national-scale operational remote sensing-based rice crop information and monitoring systems using sensors in the optical spectrum due to extensive and pervasive cloud cover and long-term rainfall limiting the acquisition capability of satellite imagery (Zhang et al., 2017; Zhou et al., 2017; Setiyono et al., 2017; Nguyen et al., 2017).

* Corresponding author
Space-borne Synthetic Aperture Radar (SAR) sensors are an effective system for monitoring crop growth, crop classification and mapping of crop area based on the crop canopy interaction of radar signals due to backscattering coefficients of the earth surface as well as its advantages of all-weather, day and night imaging, canopy penetration, and high-resolution capabilities. Time series analysis of multi-temporal SAR backscatter values is the most common data analysis approach for identification of paddy rice crop and used to retrieve the rice growing cycle based on the temporal variations in the SAR backscatter $\left(\sigma^{\circ}(\mathrm{dB})\right)$ signal, due to higher annual variation in $\sigma^{\circ}(\mathrm{dB})$ from rice fields compared to any other agricultural crop. A substantial number of studies have already been reported using medium resolution SAR data located in India, Bangladesh, Thailand, China, Vietnam, Cambodia, Japan , Indonesia, Philippines and Europe, compared to high spatial resolution SAR data $(\leq 20 \mathrm{~m})$ over a fewer locations.

C-band SAR sensors with single and multi-polarization capability is found most attractive for rice monitoring, discrimination of different growth stages and mapping at regional or continental scale, because image data from other SAR sensors in L-band and X-band due to limited spatial coverage (e.g., Terra-SAR-X) or longer revisit time (e.g., ALOS PALSAR). Multi-temporal Space-borne SAR sensor such as RADARSAT-1 data have been used to identify rice with a classification accuracy of $91 \%$. The applications of C-band SAR data with VV polarization, HV polarization, and $\mathrm{HH}$ polarization for crop classification showed better classification accuracy of $\mathrm{HH}$ polarization compared to $\mathrm{VV}$ polarization and 
HV polarization (Zhang et al., 2017; Zhou et al., 2017; Setiyono et al., 2017; Nguyen et al., 2017). Therefore, multi-polarization SAR data provides higher classification accuracy than that of single-polarization SAR data. Further, increasing the polarization mode can increase the classification accuracy, but multi-frequency SAR sensors can effectively improve the classification accuracy compared with single-frequency SAR sensor. Several investigations reveals that the C-band likepolarized ratio $(\mathrm{HH} / \mathrm{VV})$ is a useful parameter for mapping and monitoring rice cropland, whereas $\mathrm{HH} / \mathrm{VV}$ ratio is used for discriminating rice crops from bananas, forest, and water. The backscatter coefficients of cross-polarized SAR values have a significant correlation with the development of rice plants. Threshold value $(\mathrm{dB})$ of the SAR backscattered image are selected for the identification of the potential rice growing area, which depends on the changes in the magnitude of $\mathrm{VH}$ backscatter and the SAR geometry (e.g., incidence angle) and expected to vary between 8 to $9 \mathrm{~dB}$. The backscatter value of SAR images increases as the plant size increases and eventually the SAR images show no significant difference between rice fields and other agricultural fields or vegetated areas. For the reproductive stage, backscatter values continuously keep increasing until achieve the maximum value and the values of backscattering coefficient vary between the range of $-17 \mathrm{~dB}$ and $-13 \mathrm{~dB}$ depending upon the variations in incidence angle, water level in the fields, cultivation activities or the rice varieties. During the ripening phase, a slight decrease in SAR backscatter signal is observed due to the fact that the plants dry before the harvesting. The backscattering values of SAR images of paddy surfaces just before transplanting have nearly the same backscattering value of $-23.8 \mathrm{~dB}$ for water surfaces.

In this paper, knowledge based classifier have been modeled based on backscattering coefficients of Space-borne SAR sensors with rice canopy for monitoring the crop growth analysis depending upon the different radar parameters such as wavelength, incidence angle, polarization as well as its interaction with different stages of rice-crop growth.

\section{RICE GROWING AND RICE CROPPING SYSTEMS}

\subsection{Rice Growing Stages}

The temporal aspect of rice development is very important for the understanding of the radar backscattering responses of rice fields at different growing stages. The rice crop cycle usually takes 3-6 months for the harvesting depending on the variety and the climatic conditions from germination to maturity. During this period, rice completes basically two distinct sequential growth stages such as vegetative and reproductive as shown in Fig.1.

The reproductive stage is subdivided into pre-heading and postheading periods, known as the ripening period. In the tropical climate, 120 -day variety, when planted spent about 60 days in the vegetative stage, 30 days in the reproductive stage, and 30 days in the ripening stage (Nguyen Lam-Dao, 2009). Vegetative stage refers to a period from sowing-transplanting period to the initiation of heading. The vegetative stage is characterized by active tillering, gradual increase in plant height, and leaf emergence at regular intervals, which contributes to the increasing of the leaf area. Tillering starts about 15 day after sowing and continues until flowering. The reproductive stage is characterized by stem elongation, which increases plant height, decline in tiller number, and emergence of the flag leaf, booting, heading, and flowering. The growth of crop height and biomass stops after heading and the leaves change their orientation.
Ripening stage refers to the length of ripening period from heading to maturity, which is largely affected by temperature that ranges from about 30 days in the tropics to 65 days in cool, temperate regions. Ripening is characterized by leaf senescence and grain growth with a decrease of leaf and stem moisture content, and a decrease of the number of leaves.

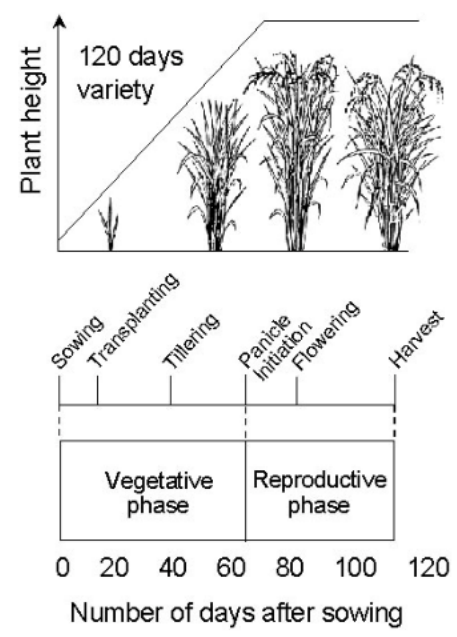

Fig.1: Rice Growing Stages

\subsection{Rice Cropping Systems}

The combination of hydrology, rainfall pattern, and availability of irrigation determines the variety of rice-based cropping systems. In general, the wetland rice production can be classified into irrigated rice fields and rain-fed rice fields. The rain-fed rice fields are irrigated by rainfall, or supplemented by localized runoff collection, whereas the water is supplied artificially from a surface or underground source for irrigated rice fields (Nguyen Lam-Dao, 2009). The rice cropping systems are also characterized by (a) size of rice field ranging from small $(0.5-1 \mathrm{ha})$ to large (b) different sowing dates from field to field (c) cultural practices (sowing, transplanting) and (d) rice varieties. The main rice cropping systems are given in Table-1

\begin{tabular}{|c|c|}
\hline $\begin{array}{c}\text { Rice cropping } \\
\text { system }\end{array}$ & Rice season \\
\hline Single rice crop & $\begin{array}{c}\text { Traditional rice (rain-fed) } \\
\text { Summer Autumn - Autumn Winter } \\
\text { (rain-fed) }\end{array}$ \\
\hline Double rice crop & $\begin{array}{c}\text { Winter Spring - Summer Autumn } \\
\text { (irrigated) }\end{array}$ \\
\hline Triple rice crop & $\begin{array}{c}\text { Winter Spring - Summer Autumn - } \\
\text { Autumn Winter }\end{array}$ \\
\hline
\end{tabular}

Table-1: Main Rice-based Cropping Systems

\section{BACKSCATTERING COEFFICIENTS OF SAR SIGNALS WITH RICE CROP-CYCLE}

Radar backscattering coefficients increases with increasing biomass till attending its saturation depending upon the radar frequency bands. In the forest environment, radar backscattering at P-band and L-band is dominated by scattering processes involving trunks and branches as the major woody biomass components, whereas, radar back-scattering at C- band and X- 
band is dominated by scattering processes in the top crown layer of branches and foliage. Radar canopy scattering measurements are found correlated with leaf-area-index (LAI), which is used for SAR vegetation interpretations. However, there is a huge challenge for the SAR data to estimate rice yield with empirical models due to complex microwave scattering mechanism of rice canopy ( Chai K., 2018; Nguyen et al.,2017; Nguyen Lam-Dao, 2009). The physical interaction of radar signals with rice canopy constituents cannot be explicitly described with simple mathematical formula.

In general, the total backscatter of rice paddies by SAR image contains (1) volume scattering in rice canopy itself (ears, leaves and stems), (2) multiple scattering between the canopy layer and underlying ground surfaces, and (3) surface scattering by the ground surfaces (soil or water). At relatively mature stage, scattering intensity of rice canopy largely depends on the scattering of rice ears besides the dominant leaves. The modeling integrates the major scattering processes in a rice canopy with several assumptions such as (a) the ground surface is a smooth surface with dielectric constant of water, since paddy fields are flooded during the growing season, (b) a rice canopy consists of three layers (i.e., ears, leaves, and stemlayers), (c) ears and stems are expressed as short cylinders, (d) leaves are expressed as narrow and long ellipses and (e) the leaf angle distribution is expressed by a specific probability distribution function.

Accordingly, the total backscattering coefficient from a Canopy $\left(\sigma_{\text {total }}\right)$ is expressed as a linear combination of volume scattering from each component, its double bounce with ground, and ground surface scattering (in power unit) (Inoue et al., 2014)

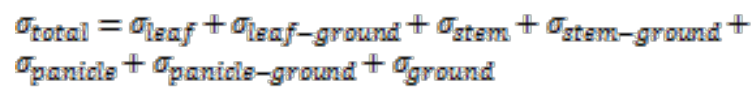

Where, the $\sigma_{\text {leaf }}, \sigma_{\text {stem }} \sigma_{\text {panicle }}$ are volume scattering of leaves, stems, and panicles, respectively. The $\sigma_{\text {leaf-ground }}$, $\sigma_{\text {stem-ground }}$ and $\sigma_{\text {panicle-ground }}$ are double bounce between each component and ground. The $\sigma_{\text {ground }} \sigma$ ground is ground surface scattering.

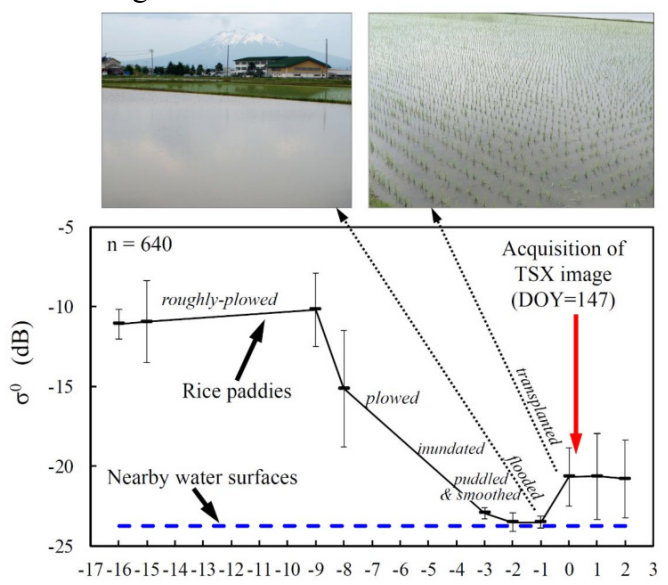

Fig.2. Days after Transplanting

Paddy fields are flooded several days before transplanting, and puddling/reveling is practiced one or two days before transplanting rice plants. The dates for the panicle initiation stage, heading stage, and maturing stage are mid-July, earlyAugust, and mid-September, respectively. The sequential change of backscattering values in rice paddies during the transplanting season revealed that backscattered signals reached the value of nearby water surfaces a day before transplanting, and increased significantly by $3 \mathrm{~dB}$ just after transplanting the rice plant. The maximum leaf area index (LAI) usually occurs around two weeks before heading stage. At the maturing stage, the number of panicles is equal to that of stems, so plenty of panicles are distributed at the top layer of a canopy. Paddy fields are irrigated continuously until the mid-maturing stage, so the soil surface of paddy fields is under flooded conditions during most growing periods. The backscattering values of SAR images of paddy surfaces just before transplanting have nearly the same backscattering value of $-23.8 \mathrm{~dB}$ for water surfaces ( Inoue et al., 2014) as shown in Fig.2

The variation of radar backscattering coefficients with growth of rice crops biomass and rice growth stage at C-Band RADARSAT and ERS SAR sensors (Nguyen et al.,2017; Nguyen Lam-Dao, 2009) are shown in Fig.3 and Fig.4 respectively.

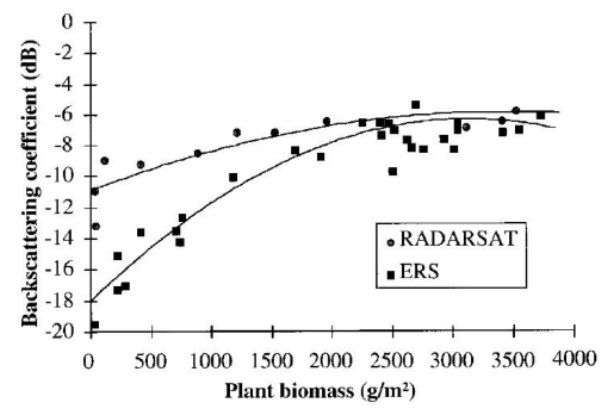

Fig.3: Variation of backscattering coefficients with rice-cropbiomass

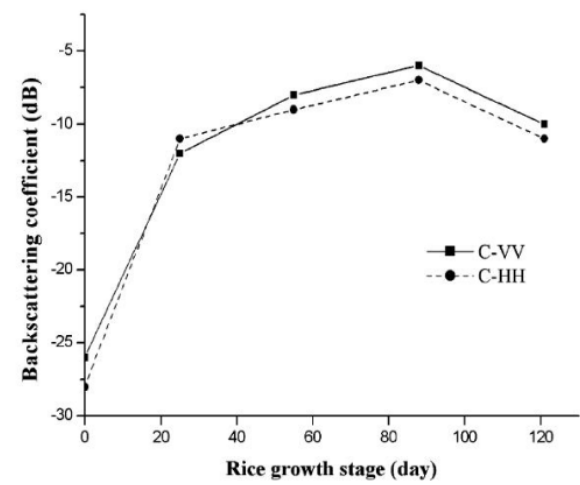

Fig.4: Variation of backscattering coefficients with rice growth

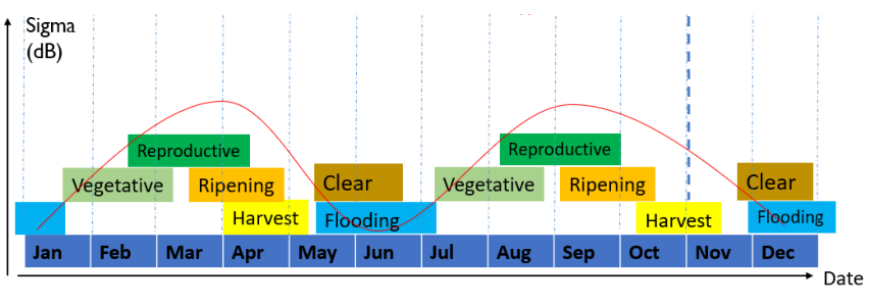

Fig.5: Sentinel-1 acquisition covers the seasonal rice crop calendar and growth stages (rice-rice)

The variation of backscattering coefficients with seasonal rice crop calendar and rice-crop growth stages is depicted in Fig.5 by the SAR sensors of Sentinel-1 ( Chai K., 2018). Similarly, backscattering dynamics of VV and VH polarized Sentinel-1 
from transplanting to harvesting stage of rice crop growth is depicted in Fig.6.

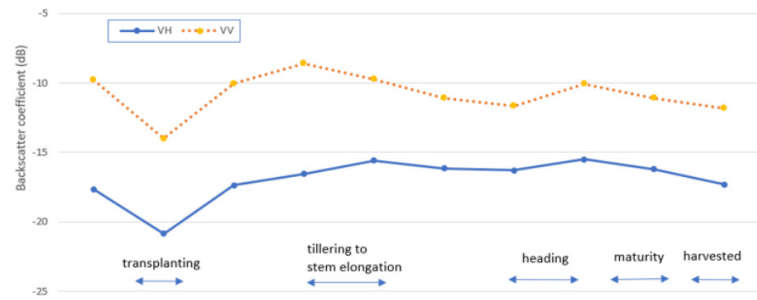

Fig.6: Backscatter dynamic of the rice plot at the VH and VV polarizations

The variation of SAR backscattering coefficients for rice crop planted fields with standing water levels after 20 days of sowing are given in the Table-2 for C-band at $\mathrm{HH}$ and $\mathrm{VV}$ signals (Nguyen et al.,2017; Nguyen Lam-Dao, 2009).

Table-2: Backscattering coefficients after 19 days of transplantation

\begin{tabular}{|c|c|r|r|}
\hline $\begin{array}{c}\text { Age } \\
(\mathbf{d a y})\end{array}$ & $\begin{array}{c}\text { Water height } \\
\mathbf{( c m )}\end{array}$ & \multicolumn{1}{c|}{$\boldsymbol{\sigma}_{\mathrm{HH}}^{\mathbf{0}}$} & \multicolumn{1}{c|}{$\boldsymbol{\sigma}_{\mathrm{VV}}^{\mathbf{0}}$} \\
\hline 19 & 7.0 & -9.1 & -14.9 \\
\hline 19 & 5.0 & -9.1 & -13.6 \\
\hline 19 & 2.0 & -7.2 & -11.6 \\
\hline 16 & no-water & -3.3 & -6.3 \\
\hline
\end{tabular}

The analysis of temporal variation of the rice parameters and radar backscattering coefficients at different rice growing stages (Nguyen Lam-Dao, 2009) can be done with the methods given in Fig.7.

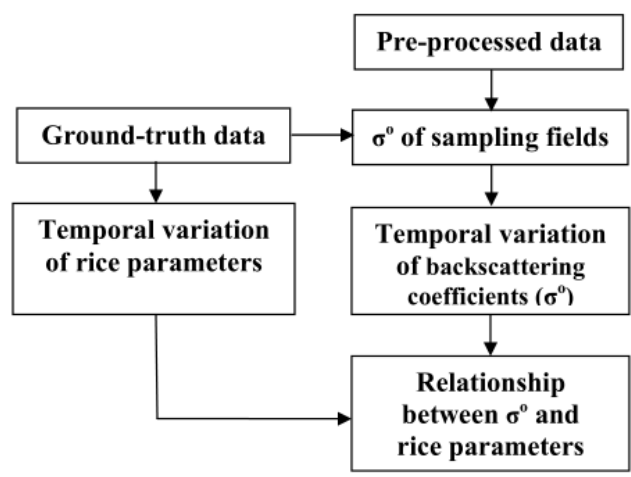

Fig.7: Methods for the analysis of radar backscatter

\section{MODELLING OF KNOWLEDGE BASED CLASSIFIER FOR RICE GROWTH MONITORING}

\subsection{Obtain usable data set (SAR images) for the area to be} examined:

a. Identify the study area where rice cultivation, pattern and harvesting time is to be predicted.

b. For example: Multitemporal Sentinel-1 image acquisition for a specific year. SAR images from Sentinel-1A IW mode can be acquired over the study area for the Dry Season and Wet Season to identify different rice cropping pattern based on a temporal analysis of the backscatter coefficient $(\mathrm{dB})$.

\subsection{SAR data pre-processing:}

(a) Apply orbit file, radiometric calibration and terrain correction: The orbit file provides accurate satellite position and velocity information. Geometric and radiometric correction is necessary for the comparison of SAR images acquired with different sensors or acquired from the same sensor but at different times, in different modes, or processed by different processors.

(b) Create stacks and time-series filtering: Stack one or more slave images with respect to a master image acquired with the same observation geometry. After stacking, the multitemporal filter can reduce the speckle noise on the timeseries images both in spatial and temporal dimension.

(c) The Digital Number values can be converted into backscatter coefficient $\left(\sigma^{\circ}\right)$ in the decibel scaling $(\mathrm{dB})$ for the analysis and classification using the equation:

$$
\sigma 0(d B)=10 \log 10(D N)
$$

(d) Two different polarizations, $\mathrm{VH}$ and $\mathrm{VV}$, and the band ratio $(\mathrm{VV} / \mathrm{VH})$ should be compared based on the temporal evolution of the backscatter coefficient over the sample plots. Select the polarization or ratio that provides the most promising significance for discriminating different crops at specific growth stages as well as representing the real rice growth cycle.

(e) Parameters definition and temporal features extraction a. After field data analysis and polarization selection, define the threshold parameters and corresponding temporal features to classify different cropping patterns.

b. $\quad$ Threshold parameters are defined from an agronomic perspective, which also required a prior knowledge of crop calendar, maturity duration, crop practices from field survey as well as the SAR temporal behaviours.

\subsection{Knowledge Based Classifier Model:}

Classifier model are based on two models: Random Forest classifier and an Image Recognition model ( Fig.8). The random forest (RF) classification algorithm belongs to an ensemble classifier class and is built on multiple decision trees, with each tree being fitted to a different bootstrapped training sample and a randomly-selected set of predictive variables. The final classification or prediction results are obtained by voting. A large number of studies have proved that the random forest algorithm has high prediction accuracy, good tolerance for abnormal values and noise, and is not prone to over-fitting.

For an image recognition model, retrained neural network will be applied using transfer learning. For example, the image recognition model called Inception V3 can be considered. It consists of two parts:

I Feature extraction part with a convolutional neural network.

ii Classification part with fully-connected and softmax layers.

iii This model will be fed with various pre-processed labelled images (different growth stages) for the training stage and will be trained till it achieves good accuracy. 


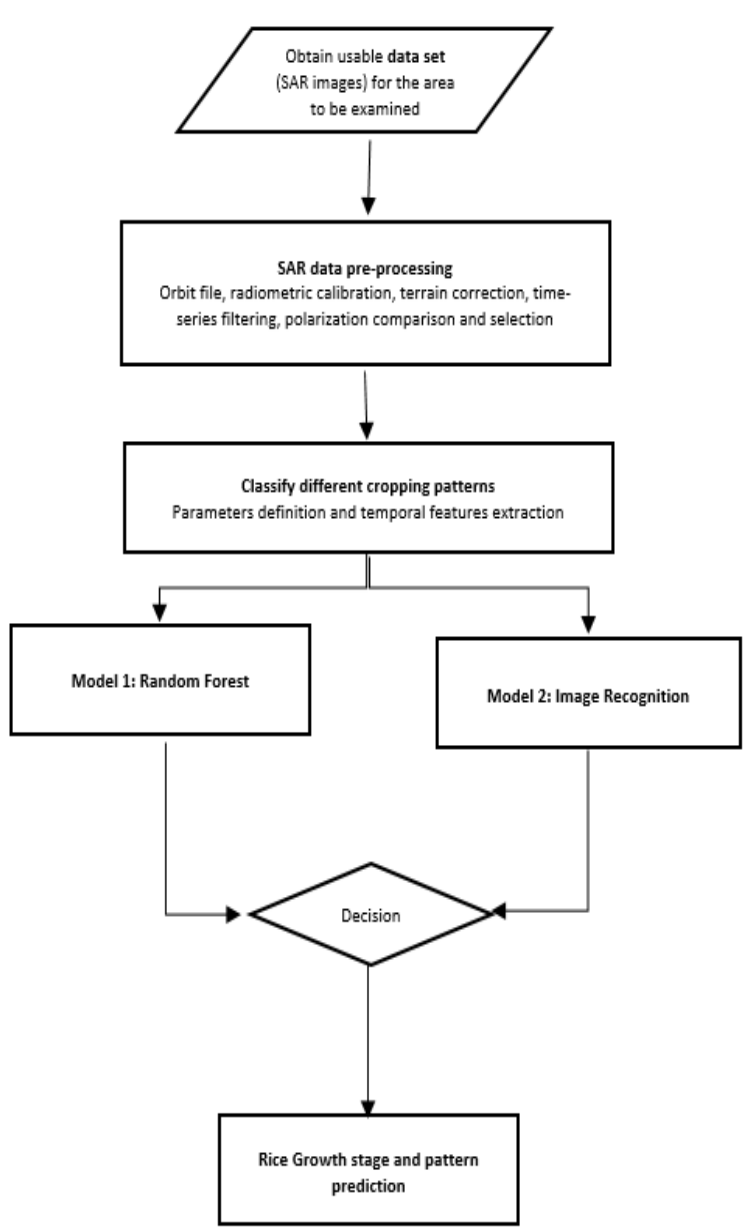

Fig.8: Flow Chart of Knowledge Based Classifier

\subsection{Prediction:}

The prediction of the final output will be made taking the decision based on the two model outputs. Both the outputs will be considered, and a threshold will decide the stage of rice growth. Based on the validation results, pattern maps will be generated.

\section{CONCLUSION}

In this paper, a new method of knowledge based classifier have been discussed for the rice growth monitoring depending upon the radar parameters as well as the earth surface characteristics parameters responsible for the growth of rice crops. The data analysis of various SAR sensors of space-borne system can be used for development of the Knowledge Based Classifier of rice crops system for monitoring the growth , mapping of cultivated area and yield prediction/ forecasting of the rice crops by validating the backscattering coefficients of SAR images with the test sites.

\section{REFERENCES}

Chai K., 2018, Detecting Rice Cropping Patterns with Sentinel1 Multi-temporal Imagery, Faculty of Geo-Information Science and Earth Observation of the University of Twente, MS Thesis, Enschede, The Netherlands, February, 2018

Inoue Y., Sakaiya E., and Wang C., 2014, Potential of X-Band Images from High-Resolution Satellite SAR Sensors to Assess
Growth and Yield in Paddy Rice, Remote Sens. 6, pp. 59956019

Nguyen D. B. and Wagner W., 2017, European Rice Cropland Mapping with Sentinel-1 Data: The Mediterranean Region Case Study, Water, 9, 392; doi:10.3390/w9060392

Nguyen Lam-Dao, 2009, Rice Crop Monitoring using New Generation Synthetic Aperture Radar (SAR) Imagery, Thesis: Doctor of Philosophy,2009, University of Southern Queensland, ,Australia

Setiyono T. D., Holecz F., Khan N. I., Barbieri M., Quicho E.,, Collivignarelli F.,Maunahan A., Gatti L., and Romuga G.C., 2017, Synthrietic Aperture Radar (SAR)-based paddy rice monitoring system: Development and application in key rice producing areas in Tropical Asia, IOP Conf. Series: Earth and Environmental Science 54, 012015, doi:10.1088/1755$1315 / 54 / 1 / 012015$

Zhang Y., Yang B., Liu X., and Wang C., 2017, Estimation of rice grain yield from dual-polarization Radarsat-2 SAR data by integrating a rice canopy scattering model and a genetic algorithm, International Journal of Applied Earth Observation and Geoinformation 57, pp. 75-85

Zhou T., Pan J., Zhang P., Wei S., and Han T., 2017, Mapping Winter Wheat with Multi-Temporal SAR and Optical Images in an Urban Agricultural Region, Sensors, 17, 1210

Wang, C., Wu, J., Zhang, Y., Pan, G., Qi, J. and Salas W.A., 2009, Characterizing L-band scattering of paddy rice in southeast China with radiative transfer model and multitemporal ALOS/PALSAR imagery. IEEE Trans. Geosci. Remote Sens. 47, pp. 988-998 